\title{
The Nature of Nonthermal X-ray Filaments Near the Galactic Center
}

\author{
F. Yusef-Zadeh \\ Dept. Physics and Astronomy, Northwestern University, 2145 Sheridan Road, \\ Evanston IL. 60208 \\ M. Wardle \\ Department of Physics, Macquarie University, Sydney, NSW 2109, Australia \\ M. Muno \\ Dept. Physics and Astronomy, UCLA, Los Angeles, CA 90095 \\ C. Law \\ Northwestern University, 2145 Sheridan Road, Evanston IL. 60208 \\ M. Pound \\ Dept of Astronomy, University of Maryland, MD 20742
}

\begin{abstract}
Recent Chandra and XMM-Newton observations reported evidence of two Xray filaments G359.88-0.08 (SgrA-E) and G359.54+0.18 (the ripple filament) near the Galactic center. The X-ray emission from these filaments has a nonthermal spectrum and coincides with synchrotron emitting radio sources. Here, we report the detection of a new X-ray feature coincident with a radio filament G359.90-0.06 (SgrA-F) and show more detailed VLA, Chandra and BIMA observations of the radio and X-ray filaments. In particular, we show that radio emission from the nonthermal filaments G359.90-0.06 (SgrA-F) and G359.54+0.18 (the ripple) has a steep spectrum whereas G359.88-0.08 (SgrA-E) has a flat spectrum. The X-ray emission from both these sources could be due to synchrotron radiation. However, given that the $20 \mathrm{~km} \mathrm{~s}^{-1}$ molecular cloud, with its intense $1.2 \mathrm{~mm}$ dust emission, lies in the vicinity of SgrA-F, it is possible that the X-rays could be produced by inverse Compton scattering of far-infrared photons from dust by the relativistic electrons responsible for the radio synchrotron emission. The production of X-ray emission from ICS allows an estimate of the magnetic field strength of $0.08 \mathrm{mG}$ within the
\end{abstract}


nonthermal filament. This should be an important parameter for any models of the Galactic center nonthermal filaments.

Key words: ISM: individual: (G359.88-0.08) (G359.88-0.08), ISM: individual: (G359.88-0.08), ISM: dust grains: Inverse Compton Scattering

\section{Background}

One of the characteristics that defines the center of our Galaxy is a significant amount of thermal and nonthermal material distributed in this region. The nonthermal emission arises mainly from a variety of synchrotron sources such as SNRs, unusual filamentary structures and a diffuse $1^{0}$-scale lobe running perpendicular to the Galactic plane (e.g., Law et al. 2004). On the other hand, thermal emission arises from stellar sources, HII regions, and the dense clouds of dust and gas pervading the inner two degrees of the Galactic center (see Morris and Serabyn and references therein 1996). One of the questions that naturally arises from these observations is whether the inverse Compton scattering (ICS) of infrared radiation by relativistic particles is an important contribution to the nonthermal X-ray emission from this region of the Galaxy. Here we investigate this possibility on a local scale and examine the application of ICS in three nonthermal filaments that are detected in both radio and $\mathrm{X}$-ray wavelengths. All the filaments lie in the vicinity of molecular clouds characterized by their high density $\approx 10^{4}-10^{5} \mathrm{~cm}^{-3}$ and high temperature $\approx$ 70 K (e.g., Morris and Serabyn 1996; Martin et al. 2004).

\section{Radio Continuum, Molecular Line and X-ray Observations}

A nonthermal X-ray source XMM J174540-2904.5 was first reported by Sakano et al./ (2003), based on 50 ks exposure Chandra and XMM-Newton observations of the Galactic center. XMM J174540-2904.5 has a hard spectrum with an extent of $\approx 15^{\prime \prime}$ and is displaced by $\approx 4^{\prime}$ south of the well-known compact radio continuum source $\mathrm{SgrA}^{*}$ at the dynamical center of the Galaxy. XMM J174540-2904.5 coincides with a nonthermal radio feature, the SgrA-E "wisp" G359.88-0.08 (Ho et al. 1985; Yusef-Zadeh and Morris 1987). Sakano et al. (2003) identify XMM J174540-2904.5 as a nonthermal radio filament with characteristics similar to those observed throughout the Galactic center (e.g., Yusef-Zadeh, Morris and Chance 1984; Morris and Serabyn 1996). Another interpretation was recently given by Lu, Wang and Lang (2003) who suggest XMM J174540-2904.5 traces a nonthermal pulsar wind nebula. 
We carried out a detailed radio and X-ray study of the SgrA-E source as well as a new source, SgrA-F, using the long exposure 600ks Chandra observation of the Galactic center (Baganoff et al. 2003; Muno et al. 2003). Radio continuum (projects AH-1, AA31 and AY131) were carried out at 20cm, 3.6 and $2 \mathrm{~cm}$ using the Very Large Array (VLA) of the National Radio Astronomy Observatory. ${ }^{1}$ SgrA-E and F sources were also imaged in the CS(2-1) transition at 97.980968 $\mathrm{GHz}$ with the Berkeley-Illinois-Maryland Association (BIMA) interferometer ${ }^{2}$ during the 2002-2003 observing season. The sources were observed with two antenna pointings centered at $(R A, D e c)_{2000.0}=\left(17^{h} 45^{m} 40 s^{s} 4,-29^{\circ} 04^{\prime} 30\right)$ and $\left(17^{h} 45^{m} 38.33,-29^{\circ} 03^{\prime} 30\right)$ with an LSR velocity of $20.0 \mathrm{~km} \mathrm{~s}^{-1}$. We observed in the $\mathrm{C}$ configuration of the array, sampling spatial frequencies from $2 \mathrm{k} \lambda$ to $29 \mathrm{k} \lambda$. Absolute flux calibration was derived from observations of Mars immediately before or after the source track. The quasar 1733-130 was used as the phase calibrator and secondary flux calibrator. We also obtained fullysampled single-dish maps covering the region imaged with the array in CS(21) using the Five Colleges Radio Astronomy Observatory (FCRAO) $14 \mathrm{~m}$ telescope, which has a $45^{\prime \prime}$ (FWHM) beam at $115 \mathrm{GHz}$. The CS single-dish data were resampled to the BIMA velocity resolution and put on the same flux scale as the BIMA observations using a conversion from antenna temperature of $43 \mathrm{Jy} / \mathrm{K}$ (M. Heyer 1999, private communication). The single-dish data were then linearly combined with the "dirty" interferometric maps and jointly deconvolved (Stanimirovic et al. 1999). The $1 \sigma$ rms noise per channel in the final combined map is $0.18 \mathrm{Jy} /$ beam. $(1 \mathrm{Jy} /$ beam $=1.1 \mathrm{~K}$ at the CS frequency and synthesized beam size).

Twelve separate pointings toward the Galactic center have been carried out using the Advanced CCD Imaging Spectrometer imaging array (ACIS-I) aboard the Chandra X-ray Observatory, in order to monitor Sgr A* (Baganoff et al. 2003) The ACIS-I is a set of four 1024-by-1024 pixel CCDs, covering a field of view of $17^{\prime} \times 17^{\prime}$. The CCDs measure the energies of incident photons, with a resolution of 50-300 eV (depending on photon energy and distance from the read-out node) within a calibrated energy band of $0.5-8 \mathrm{keV}$. The angular resolution at the aim-point of the telescope is better than $0.5^{\prime \prime}$ at $6.4 \mathrm{keV}$, while within the inner $4^{\prime}$ from the aim-point it is always better than $2^{\prime \prime}$. We obtained spectra of SgrA-E and F using the techniques described in detail in Muno et al. (2003). In brief, we created an event list for each observation in which we corrected the pulse heights of each event for the position-dependent charge-transfer inefficiency. The final total live time was $626 \mathrm{ks}$. We then ap-

1 The National Radio Astronomy Observatory is a facility of the National Science Foundation, operated under a cooperative agreement by Associated Universities, Inc.

2 The BIMA interferometer is operated under a joint agreement between the University of California, Berkeley, the University of Illinois, and the University of Maryland with support from the National Science Foundation. 
plied a correction to the absolute astrometry of each pointing using three Tycho sources detected strongly in each Chandra observation (Baganoff et al. 2003), and re-projected the sky coordinates of each event to the tangent plane at the radio position of Sgr $\mathrm{A}^{*}$ in order to produce a single composite image. The image was searched for point sources using the program wavdetect in three energy bands $(0.5-8 \mathrm{keV}, 0.5-1.5 \mathrm{keV}$, and $4-8 \mathrm{keV})$ using a significance threshold of $10^{-7}$. SgrA-E and F were both detected as part of this search, the results of which are listed in the electronic version of the catalog (Muno et al. 2003).

\section{G359.88-0.08 (SgrA-E) and G359.90-0.06 (SgrA-F)}

Figure 1 displays contours of X-ray emission from XMM J174540-2904.5 superimposed on a grayscale continuum image at $2 \mathrm{~cm}$ with a resolution $2.2^{\prime \prime} \times$ $1.3^{\prime \prime}$. The coincidence of X-ray and radio emission from the elongated source SgrA-E is clearly noted. The bright circular feature SgrA-G to the southwest corner is an HII region (Ho et al. 1985; Yusef-Zadeh and Morris 1987). The radio emission from SgrA-E extends for $\sim 2^{\prime} \times 15^{\prime \prime}$ with a peak brightness of $5.5 \mathrm{mJy}$ beam $^{-1}$ at the apex of this "banana-like" structure.

We also detect an additional X-ray source coincident with SgrA-F located to the northwest of SgrA-E in Figure 1. Figure 2 shows a close-up view of the contours of total intensity at $20 \mathrm{~cm}$ superimposed on an X-ray grayscale image of SgrA-F source. The radio image of SgrA-F consists of two parallel subfilaments, one of which is seen to have an X-ray counterpart with a size of $\approx 6 " \times 3^{\prime \prime}$ (length $\times$ width). The coincidence between radio and $\mathrm{X}$-ray emission from the fainter radio subfilament of SgrA-F is detected at $(R A, D e c)_{2000.0}=17^{\mathrm{h}} 45^{\mathrm{m}} 38^{s} .09,-29^{\circ} 03^{\prime} 20.35^{\prime \prime}$. The lack of X-ray emission from the southern peak of SgrA-F in Figure 2 is in part due to the lowsensitivity region experienced in the chip gaps of the ACIS observations. Most of the X-ray sources have a shape similar to that expected from the Chandra point spread function (PSF), whereas SgrA-F is clearly more extended than the PSF. Therefore, it seems unlikely that it is a chance association with an extended radio source. Figure 3 shows high resolution radio and X-ray images of SgrA-E. The X-ray and radio emission from SgrA-E are well-correlated, both displaying a sharp eastern edge and a diffuse western edge. The radio filament SgrA-E is much more elongated in its extent than its X-ray counterpart, these images show clear morphological evidence that the X-ray and radio emission from the brightest segment of SgrA-E arise from the same region. Similarly, although with limited X-ray data affected by the chip gap, the extended X-ray emission appears to be correlated with the fainter component of the filamentary structure SgrA-F in radio wavelengths. 
Figure 4 shows the X-ray spectra of SgrA-E and F. Both spectra are featureless, and emit copious X-ray emission between 4 and $8 \mathrm{keV}$. The X-ray spectra were modeled in XSPEC, using a power-law continuum absorbed at low energies by gas (the model phabs in XSPEC) and dust (the model dust) in the interstellar medium (Baganoff 2003). The column depth of dust was set to $\tau=0.485 \cdot N_{\mathrm{H}} /\left(10^{22} \mathrm{~cm}^{-2}\right)$, and the halo size to 10 times the PSF size. The spectrum of SgrA-E contained 3600 net counts, and is consistent with a photon index $\Gamma=2 \pm 0.5$ (where $\mathrm{I}_{\mathrm{E}} \propto \mathrm{E}^{-\alpha}$ and $\alpha=\Gamma-1$ ) power-law absorbed by $N_{\mathrm{H}}=(2.5 \pm 0.4) \times 10^{23} \mathrm{~cm}^{-2}$ (uncertainties are $90 \%$ confidence for a single parameter of interest). The total observed and unabsorbed $2-8 \mathrm{keV}$ flux from SgrA-E in the $380 \square^{\prime \prime}$ extraction region is $2.5 \times 10^{-13}$ and $1.3 \times 10^{-12} \mathrm{erg}$ $\mathrm{cm}^{-2} \mathrm{~s}^{-1}$, respectively. This corresponds to X-ray luminosity $\mathrm{L}_{X} \approx 10^{34} \mathrm{ergs}$ $\mathrm{s}^{-1}$ at the distance of $8 \mathrm{kpc}$. Assuming a bremsstrahlung continuum instead yields a similar absorption column, the best-fit value on the temperature of the emitting egion is $k T=14 \mathrm{keV}$. with a $90 \%$ lower limit $8 \mathrm{keV}$ and no upper limit.

The X-ray spectrum of SgrA-F is poorly constrained, because it is based on only 167 net counts. The $90 \%$ confidence intervals on the spectral parameters were $\Gamma=(0.8,8.2)$ and $N_{\mathrm{H}}=(1.7,7.9) \times 10^{23} \mathrm{~cm}^{-2}$. If we assume an absorption column of $2.5 \times 10^{23} \mathrm{~cm}^{-2}$, the photon index is still only constrained to $\Gamma=$ $2.0(1.1,3.0)$. The total observed and unabsorbed $2-8 \mathrm{keV}$ flux in the $30 \square^{\prime \prime}$ extraction region is $9.4 \times 10^{-15}$ and $5.7 \times 10^{-14} \mathrm{erg} \mathrm{cm}^{-2} \mathrm{~s}^{-1}$, respectively. The uncertainty on the spectral index of SgrA-F is large, but the range of values is consistent with that seen from SgrA-E.

The spectral index distribution measured at radio wavelengths shows that SgrA-E has a steeper spectrum than SgrA-F. In particular, $\alpha \approx-0.17 \pm 0.1$ is based on 2 and $6 \mathrm{~cm}$ images for SgrA-E whereas $\approx-0.80 \pm 0.1$ is measured between 6 and $3.6 \mathrm{~cm}$ for SgrA-F. The spectral index distribution between 6 and $2 \mathrm{~cm}$ was constructed by using an identical $u v$ range between 0.4 and 40 $\mathrm{k} \lambda$.

The flat spectrum of Sgr A-E suggests an interaction with a molecular cloud. Indeed, an interaction between the $20 \mathrm{~km} \mathrm{~s}^{-1}$ molecular cloud and SgrA-E and $\mathrm{F}$ has been suggested earlier in an $\mathrm{NH}_{3}$ line study of this region (Coil and Ho 2000). To examine the interaction hypothesis, high-resolution CS (2-1) observations of this region were carried out with BIMA with a spatial resolution of $20^{\prime \prime} \times 5.8^{\prime \prime}$. Figure 5 shows contours of integrated line emission between 0 and $35 \mathrm{~km} \mathrm{~s}^{-1}$ superimposed on a grayscale $6 \mathrm{~cm}$ continuum image (shown in black). In order to highlight the small-scale molecular features, in this figure we show the BIMA map without the FCRAO zero-spacing data. However, Figures 6a,b are derived from the combined data. The CS (2-1) emission appears clumpy on a scale of few arcseconds and its distribution is concentrated in the vicinity of SgrA-E and F. The most striking result 
is the recognition of a molecular filament which appears to be the counterpart to the northern extension of the banana-shaped structure of SgrA$\mathrm{E}$ near $(R A, D e c)_{2000.0}=17^{\mathrm{h}} 45^{\mathrm{m}} 39.5,-29^{\circ} 03^{\prime} 45^{\prime \prime}$. A slice cut perpendicular to the long axis of the molecular feature enveloping SgrA-E, as displayed in Figure 6a, shows that the velocities range between 10 and $40 \mathrm{~km} \mathrm{~s}^{-1}$. This position-velocity diagram indicates a velocity shift of about $5 \mathrm{~km} \mathrm{~s}^{-1}$ across the width of this molecular filament with an extent of about $15^{\prime \prime}$. This is also the region where $\mathrm{NH}_{3}$ line emission from the $20 \mathrm{~km} \mathrm{~s}^{-1}$ molecular cloud has been detected with $\mathrm{H}_{2}$ column density of $\approx 10^{24} \mathrm{~cm}^{-2}$ (Coil and Ho 2000). The CS (2-1) emission probes high density gas consistent with the high column density noted there. The brightest CS (2-1) emission, as presented by a cross in Figure 5, is adjacent to the region where X-ray emission is detected from SgrA-E. A velocity line profile of the peak position, as shown in Figure 6b, indicates a total linewidth of about $40 \mathrm{~km} \mathrm{~s}^{-1}$ from a size of about $3^{\prime \prime} \times 3^{\prime \prime}$.

Additional support for the proximity of the $20 \mathrm{~km} \mathrm{~s}^{-1}$ GMC (G359.98-0.07 or M-0.13-0.08) to SgrA-E and F comes from the distribution of dust emission. Figure 7 shows contours of the brightest $1.2 \mathrm{~mm}$ dust emission from the southern segment of the $20 \mathrm{~km} \mathrm{~s}^{-1}$ cloud (Zylka et al. 1998) superimposed on a grayscale $3.6 \mathrm{~cm}$ continuum emission from SgrA-E and F. Altogether, Figures 5 to 7 indicate that the bulk of CS $(2-1)$ emission with a velocity of $\approx 20 \mathrm{~km}$ $\mathrm{s}^{-1}$ is associated with the $20 \mathrm{~km} \mathrm{~s}^{-1}$ GMC. Moreover, there is compelling kinematic and morphological evidence from CS $(2-1)$ and $1.2 \mathrm{~mm}$ dust emission from the $20 \mathrm{~km} \mathrm{~s}^{-1}$ GMC that suggests physical interaction between the 20 $\mathrm{km} \mathrm{s}^{-1}$ cloud and SgrA-E and F.

\section{$4 \quad$ G359.54+0.18 (the ripple filament)}

The ripple filament is another isolated filamentary structure in the Galactic center region with a terminus that flares toward a HII complex. Linear polarization measurements show that the local magnetic field traces the filament (Yusef-Zadeh, Wardle and Parastaran 1997). A dense molecular cloud and an HII region are observed at one end of the filament suggesting that bending of the filament or a "ripple" is produced as a result of interaction (Bally and Yusef-Zadeh 1989; Staguhn et al. 1998). A recent 20cm radio continuum image of G359.54+0.18 shows that the length of this filament extends for about $15^{\prime}$. However, the brightest portion of the filament which is resolved into two parallel subfilaments extends only for $5^{\prime}$ (Yusef-Zadeh, Hewitt and Cotton 2004).

The detection of X-ray emission from the brightest portion of the ripple filament G359.54+0.18 was first reported by Lu, Wang and Lang (2003) as part

of the Galactic center survey carried out by Chandra. The ripple filament 
was observed nearly on-axis by Chandra (ObsID 2268) for 11-ksec. We calibrated this observation with the standard pipeline processing and analyzed with CIAO 2.2.1. A background spectrum was extracted with the same region as the source spectrum from a customized "blank-sky" file ${ }^{3}$. X-ray emission is seen from the region where the radio filament breaks up into two subfilaments. Figure 8a shows the brightest portion of G359.54+0.18 at radio and X-rays. X-ray emission from G359.54+0.18 is superimposed on a grayscale smooth Chandra image in the top panel; contours of X-ray emission superimposed on a grayscale $6 \mathrm{~cm}$ image of the northern and southern subfilaments are shown in the bottom panel. A weak X-ray filament with an extent of $1^{\prime}$ is noted exactly along the northern subfilament. The brightness temperature of the two subfilaments at $6 \mathrm{~cm}$ are similar but, interestingly, only the northern subfilament appears to have an X-ray counterpart. Figure 8b shows the weak X-ray spectrum, and the best-fit absorbed powerlaw model. The 60 count spectrum is extracted from an 11-ksec observation and is fitted with an absorbed powerlaw. The best-fit values (with $90 \%$ errors) are $N_{H}=11.4^{+14} \times 10^{22} \mathrm{~cm}^{-2}$ and $\Gamma=4.1_{-2.7}^{+4.4}$, which gives an unabsorbed 0.5 to $8 \mathrm{keV}$ flux of $3 \times 10^{-12}$ ergs $\mathrm{cm}^{-2} \mathrm{~s}^{-1}$ with upper and lower limits of $10^{-8}$ and $3 \times 10^{-13} \mathrm{ergs} \mathrm{cm}^{-2} \mathrm{~s}^{-1}$ , respectively. Note that the unabsorbed flux decreases with lower values of $\Gamma$ (i.e., a flatter spectrum). The radio flux density from the region of X-ray emission is $60 \mathrm{mJy}$ at $6 \mathrm{~cm}$ corresponding to $\nu F_{\nu} \approx 2 \times 10^{-14} \mathrm{erg} \mathrm{s}^{-1} \mathrm{~cm}^{-2}$ which is about two orders of magnitude weaker than its X-ray counterpart.

We measured spectral indices along the ripple filaments of $\alpha \approx=-0.8$ between 6 and $20 \mathrm{~cm}$ and $\approx-2$ between 6 and $3.6 \mathrm{~cm}$. The spectral index has previously been determined to be $\alpha=-0.8$ between 90 and 20cm (Anantharamaiah et al. 1991). Figure 9a,b show the spectral index distribution of an identical region of the ripple filament between $20 \& 6 \mathrm{~cm}$ and $6 \& 3.6 \mathrm{~cm}$, respectively. No noticeable difference is noted in the spectral index values of the northern and southern subfilaments. The break in the spectral index is similar to that of the "northern thread" filament G0.08+0.15 which has $\alpha \sim-0.5$ at low frequencies but steepening to $\alpha=-2$ between 6 and $2 \mathrm{~cm}$ wavelengths (Lang, Morris \& Echevarria 1999).

\section{Discussion}

\subsection{The SgrA-E Filament: Synchrotron Mechanism}

Sakano et al (2003) suggest that the X-ray emission from XMM J174540-2904 is due to synchrotron radiation emitted from $\mathrm{TeV}$ electrons. In particular,

3 see http://cxc.harvard.edu/ciao/threads/acisbackground 
they find that the X-ray spectrum from SgrA-E is consistent with steepening by 0.5 in slope between radio and X-ray wavelengths. Our improved spectral index determinations at radio and X-ray energies differs from earlier X-ray and radio spectral measurements (Sakano et al. 2003; Ho et al. 1985). On the X-ray side, Sakano et al. (2003) used older absorption cross sections and excluded the dust contribution which increases their derived absorption column by a factor of 2. Our derived photon index value is more accurately measured than earlier work by Sakano et al. (2003) who found $\Gamma=2(1.1,3.1)$. However, their best-fit power law with their uncertainties are consistent with the new fitted values, $\Gamma=2 \pm 0.5$. Since our new Chandra observations is about 20 times more sensitive than previous observations, uncertainties of the spectral fit are smaller and should be more reliable. On the radio side, we presented new high resolution radio continuum data and measured flux densities from the region with an X-ray counterpart at a number of wavelengths between 2 and $20 \mathrm{~cm}$. Our radio spectral index is -0.17 , flatter than the value of -0.4 obtained by Sakano et al. (2003) using data sets with different resolutions and $u v$ coverage.

Our radio and X-ray data show a steepening in the spectral index of $\sim 0.8$ from radio to X-rays. Such a change in the spectral index value is consistent with the synchrotron picture suggested by Sakano et al. (2003). In this picture, the elongated nature of SgrA-E which extends for more than $2^{\prime}$ compared to $30^{\prime \prime}$ extent of the X-ray filament suggests that synchrotron cooling losses are responsible for the origin of the different morphology between radio and $\mathrm{X}$ rays (see Figure 3). Assuming a magnetic field of $100 \mu \mathrm{G}$ and electron energies $\approx \mathrm{TeV}, \mathrm{X}$-ray emission has a lifetime of $\approx 10^{3}$ years which is much shorter than the lifetime of $\mathrm{GeV}$ electrons producing radio emission (e.g., Beck and Krause 2005). During this time, the diffusion of X-ray emitting particles with a velocity of $300 \mathrm{~km} \mathrm{~s}^{-1}$ can explain the $30^{\prime \prime}$ length of the filament which corresponds to about $\sim 1 \mathrm{pc}$ at the $8 \mathrm{kpc}$ distance of the Galactic center.

What could be the origin of the acceleration of particles to relativistic energies? The flat spectrum of the region traced by SgrA-E at radio frequencies provides an insight as what the cause might be. This flat spectrum is reminiscent of the W28 SNR which is known to be interacting with an adjacent molecular cloud (Dubner et al. 2002). The flat spectral index distribution is consistent with the Fermi acceleration mechanism predicting a flatter spectrum when a shock wave drives through a molecular cloud (Bykov et al. 2000). This picture is supported by the distribution and kinematics of the CS (2-1) molecular gas associated with the $20 \mathrm{~km} \mathrm{~s}^{-1}$ cloud in the vicinity of SgrA-E.

Also, we note a number of stellar X-ray sources detected in the region near SgrA-E (Muno et al. 2003). Based on the low exposure X-ray image of G359.880.08, Lu, Wang and Lang (2003) have suggested that a compact X-ray source CXOU J174539.6-290413 is located to the northern tip of XMM J1745402904.5 and that it may coincide with a pulsar responsible for producing X-ray 
and radio emission from SgrA-E. However, the deeper observations presented here (see Figure 3a) do not confirm the presence of a point-like X-ray source at the tip of the structure.

\subsection{The SgrA-F filament: synchrotron or inverse Compton emission}

Unlike the SgrA-E filament, which has flat and steep spectra in radio and X-rays, respectively, the SgrA-F filament has a steep spectrum at both radio $(\alpha \approx 0.8)$ and $\mathrm{X}$-ray wavelengths $(\alpha=\Gamma-1 \sim 1)$. In principle the radio and X-ray spectra could be segments of a single synchrotron spectrum with $\alpha \approx 0.87$ between radio and $\mathrm{X}$-rays. However, as the radio spectrum of Sgr A-F is much steeper than that of Sgr A-E, and given that the $20 \mathrm{~km} \mathrm{~s}^{-1}$ molecular cloud, with its intense $1.2 \mathrm{~mm}$ dust emission, lies in the vicinity of SgrA-F, it is possible that the X-rays could be produced by inverse Compton scattering of far-infrared photons from dust by the relativistic electrons responsible for the radio synchrotron emission.

To estimate the IC emission we adopt a greybody FIR spectrum from warm dust at temperature $T_{d}$ of the form

$$
I_{\nu}=B_{\nu}\left(T_{d}\right) \times\left(1-\exp \left(-\tau_{\nu}\right)\right)
$$

where $B_{\nu}$ is the Planck function and $\tau_{\nu}=\tau_{0}\left(h \nu / k T_{d}\right)^{\beta}$ is the dust optical depth. Then the X-ray flux at $1 \mathrm{keV}$ produced by inverse Compton scattering off a synchrotron source of flux $S_{\nu} \propto \nu^{-\alpha}$ is

$$
F_{X}=7.18 \times 10^{-19} \Gamma^{\prime}(\alpha+\beta+3) \tau_{0} \frac{T_{50}^{3+\alpha}}{B_{\mathrm{mG}}^{1+\alpha}} S_{\mathrm{mJy}} \quad \operatorname{erg~cm}{ }^{-2} \mathrm{~s}^{-1} \mathrm{keV}^{-1}
$$

where $\Gamma^{\prime}$ is the Gamma function, $S_{\mathrm{mJy}}$ is the synchrotron flux at $731 \mathrm{MHz}$, $T_{50}=T_{d} / 50 \mathrm{~K}$, and $B_{\mathrm{mG}}$ is the magnetic field strength in milligauss.

The thermal dust emission in the vicinity of SgrA-F is estimated from observations of the $20 \mathrm{~km} \mathrm{~s}^{-1}$ cloud at $1.2 \mathrm{~mm}$ with IRAM (Zylka et al. 1998), and 850 and $450 \mu \mathrm{m}$ with SCUBA (Pierce-Price et al. 2003). These measurements are on the Rayleigh-Jeans tail of the Planck function and so poorly constrain $T_{d}$ to be in the range $\sim 30-50 \mathrm{~K}$, but characterize the frequency dependence of $\tau_{\nu}$, which must scale as $\nu^{2}$ (i.e., $\beta=2$ ). The fluxes in a 36 " radius circle centered on $\mathrm{l}=359^{0} .891, \mathrm{~b}=-0.0731^{0}$ gives $\tau_{0} \approx 0.065 T_{50}$.

For a measured synchrotron flux of $25 \mathrm{mJy}$ from SgrA-F at $2 \mathrm{~cm}$ with a $\nu^{-0.75}$ spectrum, $S_{\mathrm{mJy}}=240$. For a magnetic field strength $1 \mathrm{mG}$, the estimated ICS $\mathrm{X}$-ray fluxes at $1 \mathrm{keV}$ are $8.8 \times 10^{-16}$ or $8.0 \times 10^{-17}$ erg $\mathrm{cm}^{-2} \mathrm{~s}^{-1} \mathrm{keV}^{-1}$ for $T_{d}=50 \mathrm{~K}$, and $30 \mathrm{~K}$ respectively. These nominal values are less than the 
observed flux of $3.2 \times 10^{-14} \mathrm{erg} \mathrm{cm}^{-2} \mathrm{~s}^{-1} \mathrm{keV}^{-1}$ from the SgrA-F filament, but scale as $\mathrm{B}^{-1.75}$, and match if $B \approx 0.13 \mathrm{mG}\left(\mathrm{T}_{d}=50 \mathrm{~K}\right)$ or $0.033 \mathrm{mG}$ $\left(\mathrm{T}_{d}=30 \mathrm{~K}\right)$. A recent ISO survey of the Galactic center molecular clouds suggest the presence of warm and a cold dust (Rodriguez-Fernandez et al. 2004). Most of the clouds show dust temperatures less than $40 \mathrm{~K}$. This suggests that the relatively large X-ray flux from Sgr A-F would require a low magnetic field of $0.03-0.13 \mathrm{mG}$.

The equipartition magnetic field depends on the synchrotron flux, the lowenergy cutoff of the relativistic electron energy spectrum and the depth of the source. Assuming the depth is equivalent to $10^{\prime \prime}$, the equipartition field is $0.20 \mathrm{mG}$ for a cutoff of $10 \mathrm{MeV}$ or $0.14 \mathrm{mG}$ for a cutoff of $100 \mathrm{MeV}$. Note that this assumes that no relativistic protons are present. If the depth is $100^{\prime \prime}$ which is higher than the transverse dimension of the filament, the equipartition fields are $0.11 \mathrm{mG}$ and $0.07 \mathrm{mG}$ for cutoffs of 10 and $100 \mathrm{MeV}$ respectively.

To summarize, the X-ray flux can be matched by inverse Compton emission for a dust temperature of $50 \mathrm{~K}$ and field strength $0.08 \mathrm{mG}$. This field might be in energy equipartition with the electrons provided that the depth of the source is equivalent to $100^{\prime \prime}$ (4pc at the distance of $8 \mathrm{kpc}$ ) and there are no relativistic protons present. However, almost all the Galactic center radio filaments have transverse dimensions which are typically $10^{\prime \prime}$ including the width of SgrA-F. Thus, the estimated magnetic field is sub-equipartition.

\subsection{The Ripple filament}

The kinematical and morphological arguments for the interaction of the ripple filament G359.54+0.18 with molecular gas and HII region have been given by Staguhn et al. (1998). The break in the radio spectral index of the ripple from $\alpha \sim-0.8$ to -2 implies that synchrotron emission from a single population of relativistic electrons cannot be responsible since the required radio to x-ray index is $\sim-0.85$. Using the break frequency of $5 \mathrm{GHz}$ corresponding to $6 \mathrm{~cm}$ and a magnetic field of $0.07 \mathrm{mG}$, the synchrotron lifetime of the ripple filament is $8 \times 10^{5}$ years.

Inverse Compton scattering of the FIR radiation emitted by the dust grains in the molecular clouds associated with the ripple filament does not appear to be significant: the flux predicted in this model is only comparable to the unabsorbed $0.5-10 \mathrm{keV}$ flux, $\sim 3 \times 10^{-12} \mathrm{erg} \mathrm{cm}^{-2} \mathrm{~s}^{-1}$, and even then only with the marginal assumptions that the magnetic field is $0.07 \mathrm{mG}$ and dust temperature is $50 \mathrm{~K}$. This dust temperature is similar to the hot component of dust temperature measured with ISO (Rodriguez-Fernandez et al. 2004). In addition, the X-ray emission arises only from the northern subfilament (see 
Figure 8) raising the question of why in an ICS the southern subfilament with the same radio brightness temperature and similar FIR radiation field does not have an X-ray counterpart? One explanation is variations of the magnetic field and particle density between the northern and southern subfilaments. A more detailed sensitive measurements should be important in testing this hypothesis.

We also examined the possibility that the X-ray emission may arise from synchrotron self-Compton scattering (SSC). However, the synchrotron emission from the filaments in radio wavelengths is too weak to be important in the SSC model for the following reasons: The ratio of SSC emission to inverse Compton scattering of a diluted Black Body intensity $\mathrm{W} \times B_{\nu}(T)$ is $2.5 \times 10^{-4}$ assuming that the temperature of a molecular cloud is $10 \mathrm{~K}$. The brightness temperature of $\mathrm{G} 359.54+0.18$ at $6 \mathrm{~cm}$ is $50 \mathrm{~K}$ and the synchrotron radiation spectrum depends on $\nu^{-1}$. Physically, the reason is that the synchrotron spectrum decreases at higher frequencies, whereas the Black Body spectrum increases strongly.

\section{Conclusions}

We have provided detailed X-ray and radio studies of three radio filaments near the Galactic center that have X-ray counterparts. This study suggests that two radio filaments with steep spectrum in radio wavelengths are likely to be produced by inverse Compton scattering of FIR radiation emitted from molecular clouds in the vicinity of the filaments. The inferred magnetic field strength from these measurements indicate a field strength less than $\mathrm{mG}$ associated with the filaments. However, the largest uncertainty in these estimates is the determination of dust temperature of the clouds. Future high resolution, sensitive far-IR and X-ray observations should be able to investigate the role of inverse Compton scattering in the Galactic center region which is known to have a mixture of thermal and nonthermal gas.

\section{References}

Anantharamaiah, K.R. et al. 1991, "Radio studies of the Galactic Centre. II - The arc, threads, and related features at $90 \mathrm{CM}(330 \mathrm{MHz})$ ", MNRAS, 249, 262-281

Baganoff, F.K., Maeda, Y., Morris, M., Bautz, M.W., Brandt, W.N., Cui, W. et al. 2003, "Chandra X-Ray Spectroscopic Imaging of Sagittarius A* and the Central Parsec of the Galaxy", ApJ, 591, 891-915 
Bally, J. \& Yusef-Zadeh, F. 1989, “A new system of nonthermal filaments near the Galactic center", ApJ, 336, 173-177

Beck, R. \& Krause, M. 2005, submitted to A\&A

Bykov, A.M., Chevalier, R.A., Ellison, D.C. \& Uvarov, Yu.A. 2000, "Nonthermal Emission from a Supernova Remnant in a Molecular Cloud", ApJ, 538, 203-216

Coil, A. \& Ho, P.T.P. 2000, "The Dynamics of Molecular Material within 15 PARSECS of the Galactic Center", ApJ, 533, 245-259

Dubner, G. M., Velázquez, P. F., Goss, W. M. \& Holdaway, M. A. 2002, "HighResolution VLA Imaging of the Supernova Remnant W28 at 328 and 1415 MHZ", AJ, 120, 1933-1945

Ho, P.T.P., Jackson, J.M., Barrett, A.H. \& Armstrong, J.T., 1985, "Interactions between the continuum sources in the galactic center and their immediate molecular environment", ApJ, 288, 575-579

Hüttemeister, S., Dahmen, G., Mauersberger, R., Henkel, C., Wilson, T. L. \& Martin-Pintado, J. 1998, "Molecular gas in the Galactic center region. III. Probing shocks in molecular cores", A\&A, 334, 646-658

Lang, C. Morris, M. \& Echevarria, L. 1999, "A Radio Polarimetric Study of the Galactic Center Threads", ApJ, 526, 727-743

Law, C.J., Yusef-Zadeh, F., Roberts, D.A., Hewitt, J. W., Cotton, W. D. \& Maddalena, R. J., 2004, "Multiwavelength, GBT survey of the Continuum Emission from the Inner Few Degrees of the Galactic Center", BAAS, 204, 59.02

Lu, F.G., Wang, Q.D. \& Lang, C.C. 2003, "The Chandra Detection of Galactic Center X-Ray Features G359.89-0.08 and G359.54+0.18", AJ, 126, 319-326

Martin, C.L., Walsh, W.M., Xiao, K., Lane, A. P., Walker, C. K., Stark, A. A. 2004, "The AST/RO Survey of the Galactic Center Region. I. The Inner 3 Degrees", ApJS, 150, 239-262

Morris, M. \& Serabyn, E. 1996, "The Galactic Center Environment", ARA\&A, 34, 645-702

Morris, M. \& Yusef-Zadeh, F. 1989, "The thermal, arched filaments of the radio arc near the Galactic center - Magnetohydrodynamic-induced ionization?", ApJ, 343, 703-712

Muno, M. P., Baganoff, F. K., Bautz, M. W., Brandt, W. N., Broos, P. S., et al., 2003, "X-Ray Sources with Periodic Variability in a Deep Chandra Image of the Galactic Center", ApJ, 599, 465-474

Pierce-Price, D., Richer, J.S., Greaves, J.S., , Holland, W.S., Jennes, T., Lasenby, A.N. et al. 2000, "A Deep Submillimeter Survey of the Galactic Center", ApJ, 545, L121-L125

Rodriguez-Fernandez, N.J., Martin-Pintado, J., Fuente, A. \& Wilson, T.L. 2004, "ISO observations of the Galactic center interstellar medium. Neutral gas and dust", A.\&A., 427, 217-229

Sakano, M., Warwick, R.S., Decourchelle, A. \& Predhel, P. 2003, "The discovery of a new non-thermal X-ray filament near the Galactic Centre", MNRAS, 340, 747-751 
Staghun, J., Stutzki, J., Uchida, K.I. \& Yusef-Zadeh, F. 1998, "The interaction of the Galactic center filament system G359.54+0.18 with its ambient medium", A.A., 336, 290-300

Stanimirovic, S., Staveley-Smith, L., Dickey, J. M., Sault, R. J., \& Snowden, S. L. 1999, "The large-scale HI structure of the Small Magellanic Cloud", MNRAS, 302, 417-436

Yusef-Zadeh, F., Morris, M. \& Chance, D. 1984, "Large, highly organized radio structures near the galactic centre", Nature, 310, 557-561

Yusef-Zadeh, F., Hewitt, J. \& Cotton, W. 2004, "A 20 Centimeter Survey of the Galactic Center Region. I. Detection of Numerous Linear Filaments", ApJS, 155, 421-550

Yusef-Zadeh, F. and Morris, M. 1987, "Structural details of the Sagittarius A complex - Evidence for a large-scale poloidal magnetic field in the Galactic center region", ApJ, 320, 545-561

Yusef-Zadeh, F., Wardle, M. \& Parastaran, P. 1997, "The Nature of the Faraday Screen toward the Galactic Center Nonthermal Filament G359.54+0.18", ApJ, 475, L119-L223

Zylka, R., Philipp, S., Duschl, W.J. Mezger, Herbst, T. \& Tuffs, R. 1998, "NIR and mm mosiacs of the central 100 pc", IAU Symp. 184, The Central Regions of the Galaxy and Galaxies, ed: Y. Sofue (Dordrecht:Kluwer), 291293 
Fig. 1. Contours of X-ray emission from XMM J174540-2904.5 superimposed on a grayscale $2 \mathrm{~cm}$ continuum image of the SgrA-E, F and $\mathrm{G}$ sources with a resolution of $2.27^{\prime \prime} \times 1.23^{\prime \prime}\left(\mathrm{PA}=-2^{0}\right)$. Contour levels are set at $(2,3,4,6,8,12,16,20) \times$ $1.510^{-10} \mathrm{Jy} \mathrm{beam}^{-1}$. The rms noise in the radio and X-ray images are $0.12 \mathrm{mJy}$ beam $^{-1}$ and $1.8 \times 10^{-11} \mathrm{Jy}_{\text {beam }}{ }^{-1}$, respectively.

Fig. 2. Contours of $20 \mathrm{~cm}$ emission from SgrA-F with a resolution of $2.17^{\prime \prime} \times 1.05^{\prime \prime}$ $\left(\mathrm{PA}=9.7^{0}\right)$ are superimposed on a grayscale X-ray image (in black). The contour levels are $-2,2,3,4,5,6,7,8,9,10$ mJy beam $^{-1}$ with rms noise 0.4 mJy beam $^{-1}$.

Fig. 3. (a) A full resolution X-ray image of Sgr A-E [top panel]. (b) A 2cm image of an identical region to (a) is shown on the bottom panel. Both images have a resolution of $2.27^{\prime \prime} \times 1.23^{\prime \prime}$

Fig. 4. The X-ray spectra of SgrA-E and F and their corresponding residuals. Both spectra are featureless and are best fit by an absorbed power law.

Fig. 5. Contours of velocity integrated CS (2-1) line emission between 0 and $35 \mathrm{~km}$ $\mathrm{s}^{-1}$ superimposed on a $6 \mathrm{~cm}$ radio continuum image in black. The cross indicates the region corresponding to the spectrum in Fig. $6 \mathrm{~b}$. The molecular line data does not contain the single-dish spacings.

Fig. 6. (a) A grayscale distribution of position-velocity diagram of CS (2-1) line emission is shown on the top panel. (b) A velocity profile of peak CS (2-1) emission from the cross drawn on on Figure 5 is shown on the bottom panel. Both figures include single-dish spacings.

Fig. 7. Contours of $1.2 \mathrm{~mm}$ emission from the northern peak of the $20 \mathrm{~km}$ $\mathrm{s}^{-1}$ molecular cloud $\mathrm{M}-0.13-0.08$ set at $(6,6.5,7, . .10,11, . .18,20,22,24,28$, $32) \times 0.16 \mathrm{Jy}$ (Zylka et al. 1998) with the resolution of $10.6^{\prime \prime}$. The grayscale $3.6 \mathrm{~cm}$ image (in black) shows SgrA-E, F and G sources with the resolution of $2.4^{\prime \prime} \times 6.6^{\prime \prime}$ $\left(\mathrm{PA}=-0.9^{0}\right)$

Fig. 8. (a) (top panel) An adaptively smooth X-ray image of the filament with the corresponding contours. (bottom panel) Contours of X-ray emission superimposed on a continuum image of the brightest portion of G359.54+0.18 at 6cm in J2000 coordinates. (b) The Chandra X-ray spectrum of the ripple filament with an absorbed powerlaw fit.

Fig. 9. ((a) top panel) The spectral index distribution between 20 and $6 \mathrm{~cm}$ whereas the bottom panel (b) shows the spectral index between 6 and $3.6 \mathrm{~cm}$. Both figures have selected the range of $u v$ visibilities between 1 and $30 \mathrm{k} \lambda$, a final spatial resolution of $9.5 \square^{\prime \prime}$. The cutoff values to determine the spectral index distribution in (a) are 2.2 and $0.41 \mathrm{mJy}$ beam $^{-1}$ at 20 and $6 \mathrm{~cm}$, respectively. Similarly, for (b) the cutoff values are 0.41 and $0.24 \mathrm{mJy}^{\mathrm{beam}}{ }^{-1}$ at 6 and $3.6 \mathrm{~cm}$. These cut-off values are three times the rms noise of each of the images used to construct spectral index distribution. 
This figure "f1.jpg" is available in "jpg" format from: http://arxiv.org/ps/astro-ph/0502260v1 
This figure "f2.jpg" is available in "jpg" format from: http://arxiv.org/ps/astro-ph/0502260v1 
This figure "f3a.jpg" is available in "jpg" format from: http://arxiv.org/ps/astro-ph/0502260v1 
This figure "f3b.jpg" is available in "jpg" format from: http://arxiv.org/ps/astro-ph/0502260v1 
This figure "f4.jpg" is available in "jpg" format from: http://arxiv.org/ps/astro-ph/0502260v1 
This figure "f5.jpg" is available in "jpg" format from: http://arxiv.org/ps/astro-ph/0502260v1 
This figure "f6a.jpg" is available in "jpg" format from: http://arxiv.org/ps/astro-ph/0502260v1 
This figure "f6b.jpg" is available in "jpg" format from: http://arxiv.org/ps/astro-ph/0502260v1 
This figure "f7.jpg" is available in "jpg" format from: http://arxiv.org/ps/astro-ph/0502260v1 
This figure "f8a.jpg" is available in "jpg" format from: http://arxiv.org/ps/astro-ph/0502260v1 
This figure "f8b.jpg" is available in "jpg" format from: http://arxiv.org/ps/astro-ph/0502260v1 
This figure "f9a.jpg" is available in "jpg" format from: http://arxiv.org/ps/astro-ph/0502260v1 
This figure "f9b.jpg" is available in "jpg" format from: http://arxiv.org/ps/astro-ph/0502260v1 